\title{
REGULARLY AND SINGULARLY PERTURBED CRACKS
}

\author{
BY
}

CHIEN H. WU

University of Illinois at Chicago

\begin{abstract}
In a nondimensionalized rectangular Cartesian coordinate system $\left(x_{1}, x_{2}\right)$ let $x_{2}=\varepsilon Y_{ \pm}\left(x_{1}\right)$ denote the upper and lower surfaces of a hole where $\left|x_{1}\right| \leq 1$ and $\varepsilon$ is a small parameter. As $\varepsilon$ tends to zero, the hole degenerates into a crack of length 2 . The functions $Y_{ \pm}$, together with their derivatives, are continuous and $Y_{+}-Y_{-} \geq 0$. For $\varepsilon$ not equal to zero, the hole is called a regularly (singularly) perturbed crack if $Y_{+}^{\prime}( \pm 1)=Y_{-}^{\prime}( \pm 1) \quad\left(Y_{+}^{\prime}( \pm 1) \neq Y_{-}^{\prime}( \pm 1)\right)$. Regular perturbation procedures are applied to obtain the stress intensity factors existing at the tips of regularly perturbed cracks. It is shown that the second term of a two-term expansion is not always of the order of $\varepsilon$. The notch-tip singularity associated with a singularly perturbed crack is obtained by the method of matched asymptotic expansions.
\end{abstract}

Introduction. Let $\left(x_{1}, x_{2}\right)$ be a dimensionless rectangular Cartesian coordinate system. The upper and lower surfaces of a hole may be conveniently defined by

$$
x_{2}=\varepsilon Y_{ \pm}\left(x_{1}\right) \quad\left(\left|x_{1}\right|<1\right),
$$

where

$$
Y_{+}\left(x_{1}\right)-Y_{-}\left(x_{1}\right) \geq 0
$$

and $\varepsilon$ is a small parameter. As $\varepsilon$ tends to zero, the hole degenerates into a crack of length 2. It is for this reason that the hole is referred to as a perturbed crack.

The hole is called a regularly perturbed crack if

$$
Y_{+}^{\prime}( \pm 1)-Y_{-}^{\prime}( \pm 1)=0
$$

which ensures the existence of cusps at $x_{1}= \pm 1$. For such cases the associated stress intensity factors are of prime importance. Holes with cuspidal points have been treated as stress raisers by many researchers (Westman [14], Panasyuk and Berezhnitskii [9], Berezhnitskii and Sadivskii [1, 2], and Wu [15, 16]). For the case where the cusp is formed by curved boundaries, the well-known crack-tip analysis (Williams [13]) must be modified (Ting [11]), and the addition of logarithmic terms is very often encountered.

A curvilinear crack is simply defined by $Y_{ \pm}=Y$. Other than the case of a circular-arc crack (Muskhelishvili [8]), exact solutions to curvilinear crack problems

Received June 16, 1992.

(C)1994 Brown University 
are practically nonexistent. Perturbation methods have been applied in analyzing slightly curved and kinked cracks (Goldstein and Salganik [6]; Cotterell and Rice [4]). In terms of the representation (1.1), $\varepsilon$ is the perturbation parameter and the results given in the references were accurate to the order of $\varepsilon$. Such a first-order perturbation solution has been found to be unsatisfactory for many symmetry and loading conditions. Second- and higher-order perturbation solutions are systematically introduced in this paper.

The perturbation procedure used for analyzing a regularly perturbed crack is a regular one in that all asymptotic expressions are uniformally valid throughout the region of interest. For the case of singularly perturbed cracks, which are defined by

$$
Y_{+}^{\prime}( \pm 1) \neq Y_{-}^{\prime}( \pm 1)
$$

the singularities at $x_{1}= \pm 1$ are of the notch type, which is fundamentally different from a cusp. Singular perturbation methods are applied to such singularly perturbed cracks in this paper. It is shown that the "strength" of the notch-tip singularity can be explicitly determined. Similar analysis has been successfully performed by us on stress concentration problems ( $\mathrm{Wu}[17])$.

2. Complex variable formulation. Let $a$ be a length parameter that is to be identified with a half-crack length and $\mu$ the shear modulus. We shall use $a$ and $\mu a^{2}$ as the length and force scales throughout this paper, so that all quantities are nondimensionalized relative to these parameters.

We consider plane elasticity problems in the dimensionless $\left(x_{1}, x_{2}\right)$-plane and shall use a complex formulation in terms of the dimensionless complex variable $z=x_{1}+i x_{2}$. The dimensionless displacements $u_{\alpha}\left(x_{1}, x_{2}\right)$ and stresses $\tau_{\alpha \beta}\left(x_{1}, x_{2}\right)$ may be written in terms of two complex functions $W(z)$ and $w(z)$, viz.

$$
\begin{gathered}
2\left(u_{1}+i u_{2}\right)=\kappa W(z)-z \overline{W^{\prime}(z)}-\overline{w(z)}, \\
\tau_{11}+\tau_{22}=2\left[W^{\prime}(z)+\overline{W^{\prime}(z)}\right], \\
\tau_{22}-i \tau_{12}=W^{\prime}(z)+\overline{W^{\prime}(z)}+\overline{w^{\prime}(z)}, \\
i R=i \int\left[\tau_{\beta 1} n_{\beta}+i \tau_{\beta 2} n_{\beta}\right] d s=W(z)+z \overline{W^{\prime}(z)}+\overline{w(z)},
\end{gathered}
$$

where $R=R_{1}+i R_{2}$ is the resultant force over an arc. In the preceding equations and throughout this paper prime denotes complex differentiation; $W^{\prime}$ and $w^{\prime}$ are holomorphic functions and

$$
\kappa= \begin{cases}3-4 \nu & \text { plane stress, } \\ (3-\nu) /(1+\nu) & \text { plane strain, }\end{cases}
$$

where $\nu$ is Poisson's ratio.

For the class of problems to be discussed in this paper it is more convenient to employ a third holomorphic function $f(z)$ defined by

$$
f(z)=W(z)-z \overline{W^{\prime}(\bar{z})}-\overline{w(\bar{z})} .
$$


Using Eq. (2.6) to eliminate $w$ from Eqs. (2.1)-(2.4), we get (England [5])

$$
\begin{gathered}
2\left(u_{1}+i u_{2}\right)=\kappa W(z)-W(\bar{z})+(\bar{z}-z) \overline{W^{\prime}(z)}+f(\bar{z}), \\
\tau_{22}-i \tau_{12}=W^{\prime}(z)+W^{\prime}(\bar{z})+(z-\bar{z}) \overline{W^{\prime \prime}(z)}-f^{\prime}(\bar{z}), \\
i R=W(z)+W(\bar{z})+(z-\bar{z}) \overline{W^{\prime}(z)}-f(\bar{z}) .
\end{gathered}
$$
that

The elastic infinite plane is loaded at infinity by constant stresses $\tau_{\alpha \beta}=\sigma_{\alpha \beta}$, so

$$
\begin{gathered}
W^{\prime}(z)=C_{1}-C_{-1} z^{-2}+\cdots, \\
w^{\prime}(z)=c_{1}-c_{-1} z^{-2}+\cdots \\
f^{\prime}(z)=-\bar{c}_{1}-\left(C_{-1}+\bar{C}_{-1}-\bar{c}_{-1}\right) z^{-2}+\cdots, \\
2 W^{\prime}-f^{\prime}=\left(\sigma_{22}-i \sigma_{12}\right)-\left(C_{-1}-\bar{C}_{-1}+\bar{c}_{-1}\right) z^{-2}+\cdots,
\end{gathered}
$$

as $z \rightarrow \infty$ and

$$
C_{1}=\frac{1}{4}\left(\sigma_{11}+\sigma_{22}\right), \quad c_{1}=\frac{1}{2}\left(\sigma_{22}-\sigma_{11}\right)+i \sigma_{12} .
$$

This infinite plane is also assumed to have a single traction-free cavity with its upper and lower boundaries given by

$$
z=Z_{ \pm}=x_{1}+i \varepsilon Y_{ \pm}\left(x_{1}\right) \quad\left(\left|x_{1}\right|<1\right)
$$

where $\varepsilon$ is a small dimensionless parameter. As $\varepsilon$ tends to zero, the cavity tends to the simple geometry of a linear crack of length 2 . It is clear that the functions $Y_{ \pm}\left(x_{1}\right)$ play a significant role in the analysis to follow. Physical considerations require that

$$
Y_{+}\left(x_{1}\right)-Y_{-}\left(x_{1}\right) \geq 0 \text {. }
$$

We also assume that $Y_{ \pm}^{\prime}$ are continuous so that no additional singularities, other than the ones at $x_{1}= \pm 1$, are present. With the above conditions in mind we are led to divide the class of problems into the following categories:

regularly perturbed cracks

$$
Y_{+}^{\prime}( \pm 1)=Y_{-}^{\prime}( \pm 1)
$$

singularly perturbed cracks

$$
Y_{+}^{\prime}(-1) \neq Y_{-}^{\prime}(-1) \quad \text { and } / \text { or } \quad Y_{+}^{\prime}(+1) \neq Y_{-}^{\prime}(+1) .
$$

It is clear that the singularities at $x_{1}= \pm 1$ for (2.18) are not of the crack-tip type.

The assumed traction-free condition along the cavity boundary may be integrated once to become the vanishing of $R$. Substituting Eq. (2.15) into Eq. (2.9), we have

$$
W\left(Z_{ \pm}\right)+W\left(\bar{Z}_{ \pm}\right)+\varepsilon i 2 Y_{ \pm}\left(x_{1}\right) \overline{W^{\prime}\left(Z_{ \pm}\right)}-f\left(\bar{Z}_{ \pm}\right)=0 .
$$

The determination of $W$ and $f$ subjected to the loading conditions (2.10), (2.12), and (2.19) is the underlying mathematical problem to be solved.

\footnotetext{
${ }^{1}$ For cases where the tips are smooth, a different type of boundary-layer solution could be constructed but is not considered here.
} 
Let $W(z ; \varepsilon)$ and $f(z ; \varepsilon)$ be the desired solution. If we formally set $\varepsilon$ to zero in all the relevant expressions, then it can easily be seen that all the conditions are formally satisfied by the well-known crack solution:

$$
\begin{gathered}
f(z ; 0)=f_{0}(z)=\left[\frac{1}{2}\left(\sigma_{11}-\alpha_{12}\right)+i \sigma_{12}\right] z, \\
2 W(z ; 0)-f(z ; 0)=2 W_{0}(z)-f_{0}(z)=\left(\sigma_{22}-i \sigma_{12}\right)\left(z^{2}-1\right)^{1 / 2} .
\end{gathered}
$$

This solution is termed the reduced solution or the solution to the reduced problem.

In the case that $Y_{ \pm}\left(x_{1}\right)$ satisfy Eq. (2.17), the above reduced solution is a uniformly valid zeroth-order approximation to the true solution for small but nonzero $\varepsilon$. A refined solution may therefore be built upon the reduced solution via a regular perturbation procedure. In the case that Eq. (2.17) is not satisfied, a special notchtip expansion of the Williams's type (Williams [13]) must be separately constructed. The coefficients involved in this expansion are then determined by the principle of matching (Cole [3]; Van Dyke [12]). A parallel situation dealing with stress and notch-stress concentrations induced by shallow depressions may be found in a recent result by $\mathrm{Wu}[17]$.

For regularly perturbed cracks, the associated dimensionless stress intensity factors may be computed from

$$
K_{1}-i K_{2}=\lim _{z \rightarrow \pm 1} 2 \sqrt{2 \pi}(z \mp 1)^{1 / 2} W^{\prime}(z) e^{-i \alpha_{c}^{ \pm} / 2}
$$

where $\alpha_{c}^{ \pm}$defines the crack-tip orientation and

$$
\alpha_{c}^{ \pm}=\operatorname{Tan}^{-1}\left(\varepsilon Y_{+}^{\prime}( \pm 1)\right)=\operatorname{Tan}^{-1}\left(\varepsilon Y_{-}^{\prime}( \pm 1)\right) .
$$

3. Regularly perturbed cracks. The main objective of this section is to find the first two nonzero terms of an asymptotic expansion in $\varepsilon$ for a desired stress intensity factor, with the first term being the corresponding stress intensity factor associated with the reduced solution. We shall see that the second term is not always of the order of $\varepsilon$.

In view of Eq. (2.19), the desired expansion for $W$ and $f$ may be expressed in powers of $\varepsilon$ as follows:

$$
\begin{aligned}
W(z ; \varepsilon) & \sim \sum_{n=0}^{\infty} \frac{\varepsilon^{n}}{n !} W_{n}(z), \\
f(z ; \varepsilon) & \sim \sum_{n=0}^{\infty} \frac{\varepsilon^{n}}{n !} f_{n}(z) .
\end{aligned}
$$

Substituting Eqs. (3.1), (3.2) into Eq. (2.19), we obtain

$$
\begin{aligned}
& W_{0}^{ \pm}+W_{0}^{\mp}-f_{0}^{\mp}=0 \text {, } \\
& W_{p}^{ \pm}+W_{p}^{\mp}-f_{p}^{\mp}+p ! \sum_{n=0}^{p-1} \frac{\left(i Y_{ \pm}\right)^{p-n}}{n !(p-n) !}\left[W_{n}^{(p-n)^{ \pm}}+(-1)^{p-n} W_{n}^{(p-n)^{\mp}}\right. \\
& \left.-2(p-n)(-1)^{p-n}{W_{n}^{\overline{(p-n)^{ \pm}}}}^{ \pm}(-1)^{p-n} f_{n}^{(p-n)^{\mp}}\right]=0,
\end{aligned}
$$


where the last equation applies for $p \geq 1$, and

$$
\left.W_{n}^{(p-n)^{ \pm}} \equiv \frac{\partial^{p-n}}{\partial z^{p-n}} W_{n}(z)\right|_{z=x_{1} \pm i 0}
$$

In deriving the above equations for a general pair of $Y_{ \pm}$, the interpretation of $W\left(\bar{Z}_{ \pm}\right)$needs to be clarified. Take, for example, the case $Y_{+}=Y_{-}=Y>0$. We have

$$
\begin{aligned}
& W\left(\bar{Z}_{+}\right)=W\left(x_{1}-i \varepsilon Y\right), \\
& W\left(\bar{Z}_{-}\right)=\left\{\text {analytic continuation of } W\left(Z_{+}\right) \text {on a Riemann surface }\right\}_{x_{1}-i \varepsilon Y},
\end{aligned}
$$
and hence, on letting $\varepsilon \rightarrow 0$,

$$
\begin{aligned}
& W\left(\bar{Z}_{+}\right)=W\left(x_{1}-i 0\right)=W^{-}\left(x_{1}\right), \\
& W\left(\bar{Z}_{-}\right)=W\left(x_{1}+i 0\right)=W^{+}\left(x_{1}\right) .
\end{aligned}
$$

Forming the difference and sum of the \pm versions of Eq. (3.3), we get

$$
\begin{gathered}
f_{0}^{+}-f_{0}^{-}=0, \\
\left(2 W_{0}-f_{0}\right)^{+}+\left(2 W_{0}-f_{0}\right)^{-}=0,
\end{gathered}
$$

which, together with the conditions at infinity, lead to the solutions (2.20) and (2.21), i.e.,

$$
\begin{gathered}
f_{0}(z)=-\bar{c}_{1} z=\left[\frac{1}{2}\left(\sigma_{11}-\sigma_{22}\right)+i \sigma_{12}\right] z, \\
2 W_{0}(z)-f_{0}(z)=\left(\sigma_{22}-i \sigma_{12}\right) X(z), \quad X=\left(z^{2}-1\right)^{1 / 2} .
\end{gathered}
$$

For $p=1$, Eq. (3.4) yields

$$
W_{1}^{ \pm}+W_{1}^{\mp}-f_{1}^{\mp}+i Y_{ \pm}\left[W_{0}^{ \pm}-W_{0}^{\mp}+\overline{2 W_{0}^{\prime \pm}}+f_{0}^{\prime}\right]=0
$$

which, after applying Eqs. (3.8) and (3.9), becomes

$$
W_{1}^{ \pm}+W_{1}^{\mp}-f_{1}^{\mp}=i Y_{ \pm}\left(x_{1}\right)\left[\left(\sigma_{22}-\sigma_{11}\right) \pm \frac{2 \sigma_{12} x_{1}}{\left(1-x_{1}^{2}\right)^{1 / 2}}\right] \text {. }
$$

Forming the difference and sum of the \pm versions of the above, we obtain

$$
\begin{gathered}
f_{1}^{+}-f_{1}^{-}=i\left(\sigma_{22}-\sigma_{11}\right)\left[Y_{+}\left(x_{1}\right)-Y_{-}\left(x_{1}\right)\right]+i 2 \sigma_{12} \frac{x_{1}}{\left(1-x_{1}^{2}\right)^{1 / 2}}\left[Y_{+}\left(x_{1}\right)+Y_{-}\left(x_{1}\right)\right] \\
\left(2 W_{1}-F_{1}\right)^{+}+\left(2 W_{1}-F_{1}\right)^{-}=i\left(\sigma_{22}-\sigma_{11}\right)\left[Y_{+}\left(x_{1}\right)+Y_{-}\left(x_{1}\right)\right] \\
+i 2 \sigma_{12} \frac{x_{1}}{\left(1-x_{1}^{2}\right)^{1 / 2}}\left[Y_{+}\left(x_{1}\right)-Y_{-}\left(x_{1}\right)\right]
\end{gathered}
$$

Anticipating that the second nonzero term for a stress intensity factor is not always of the order of $\varepsilon$, we also develop the appropriate boundary conditions for $W_{2}$ and $f_{2}$. The explicit form of Eq. (3.4) for $p=2$ is

$$
\begin{aligned}
W_{2}^{ \pm} & +W_{2}^{\mp}-f_{2}^{\mp}-Y_{ \pm}^{2}\left[W_{0}^{\prime \prime}+W_{0}^{\prime \prime \mp}-4 \overline{W_{0}^{\prime \prime} \pm}-f_{0}^{\prime \prime}\right] \\
& +i 2 Y_{ \pm}\left[W_{1}^{\prime \pm}-W_{1}^{\prime \mp}+2 \overline{W_{1}^{\prime \pm}}+f_{1}^{\mp \mp}\right]=0 .
\end{aligned}
$$


Applying Eqs. (3.8) and (3.9) and forming the difference and sum of the \pm versions of (3.14), we arrive at the following Cauchy and Hilbert conditions:

$$
\begin{aligned}
f_{2}^{+}-f_{2}^{-}= & -\frac{2 i\left(\sigma_{22}+i \sigma_{12}\right)}{\left(1-x_{1}^{2}\right)^{3 / 2}}\left(Y_{+}^{2}+Y_{-}^{2}\right) \\
& -i\left\{\left(Y_{+}+Y_{-}\right)\left[\left(2 W_{1}^{\prime}-f_{1}^{\prime}\right)^{+}-\left(2 W_{1}^{\prime}-f_{1}^{\prime}\right)^{-}\right]+\left(Y_{+}-Y_{-}\right)\left(f_{1}^{\prime+}+f_{1}^{\prime-}\right)\right. \\
& +2 Y_{+}\left[\left(2 \bar{W}_{1}^{\prime+}-\bar{f}_{1}^{\prime+}\right]-2 Y_{-}\left[\left(2 \bar{W}_{1}^{\prime-}-\bar{f}_{1}^{\prime-}\right)+\bar{f}_{1}^{\prime-}\right]\right\}
\end{aligned}
$$

$$
\begin{aligned}
\left(2 W_{2}-f_{2}\right)^{+} & +\left(2 W_{2}-f_{2}\right)^{-}=-\frac{2 i\left(\sigma_{22}+i \sigma_{12}\right)}{\left(1-x_{1}^{2}\right)^{3 / 2}}\left(Y_{+}^{2}-Y_{-}^{2}\right) \\
-i\left\{\left(Y_{+}-\right.\right. & \left.Y_{-}\right)\left[\left(2 W_{1}^{\prime}-f_{1}^{\prime}\right)^{+}-\left(2 W_{1}^{\prime}-f_{1}^{\prime}\right)^{-}\right]+\left(Y_{+}+Y_{-}\right)\left(f_{1}^{\prime+}+f_{1}^{\prime-}\right) \\
& \left.+Y_{+}\left[2\left(2 \overline{W_{1}^{\prime}}+{\overline{f^{\prime}}}_{1}^{+}\right)+2{f_{1}^{\prime+}}^{+}\right]+Y_{-}\left[2\left(2{\overline{W^{\prime}}}_{1}^{-}-{\overline{f^{\prime}}}_{1}^{-}\right)+2{\overline{f^{\prime}}}_{1}^{-}\right]\right\} .
\end{aligned}
$$

Curvilinear cracks $\left(Y_{+}=Y_{-}\right)$. This is essentially the class of problems considered by Cotterell and Rice [4]. Let us begin by defining

$$
Y_{ \pm}=Y\left(x_{1}\right)=\left(1-x_{1}^{2}\right) P\left(x_{1}\right)
$$

where $P\left(x_{1}\right)$ is a polynomial, so that the crack configuration is still fairly general. Substituting Eq. (3.17) into Eqs. (3.12) and (3.13), we have

$$
\begin{gathered}
f_{1}^{+}-f_{1}^{-}=i 4 \sigma_{12} x_{1} P\left(x_{1}\right)\left(1-x_{1}^{2}\right)^{1 / 2}, \\
\left(2 W_{1}-f_{1}\right)^{+}-\left(2 W_{1}-f_{1}\right)^{-}=i 2\left(\sigma_{22}-\sigma_{11}\right)\left(1-x_{1}^{2}\right) P\left(x_{1}\right) .
\end{gathered}
$$

The solution to Eqs. (3.18), (3.19) may easily be written down by inspection, viz.

$$
\begin{gathered}
f_{1}(z)=2 \sigma_{12}\left[z P(z)\left(z^{2}-1\right)^{1 / 2}-h_{1}(z)\right], \\
2 W_{1}-f_{1}=i\left(\sigma_{22}-\sigma_{11}\right)\left[\left(1-z^{2}\right) P(z)-\left(z^{2}-1\right)^{1 / 2} H_{1}(z)\right],
\end{gathered}
$$

where $h_{1}$ and $H_{1}$ are polynomials chosen so that both $f_{1}^{\prime}$ and $2 W_{1}^{\prime}-f_{1}^{\prime}$ tend to $O\left(1 / z^{2}\right)$ as $z$ tends to infinity. It can easily be checked that the second significant term in $K_{1}$ can be at most of the order of $\varepsilon^{2}$ if $\sigma_{12}=0$. Similarly, the second significant term in $K_{2}$ can be at most of the order of $\varepsilon^{2}$ if $\sigma_{11}=\sigma_{22}=0$. Thus, the inclusion of the $\varepsilon^{2}$-terms in the desired expansion is both important and useful.

Substituting all the known relations into Eqs. (3.15) and (3.16), we get

$$
\begin{gathered}
f_{2}^{+}-f_{2}^{-}=i\left(1-x_{1}^{2}\right)^{1 / 2}\left[-4 \sigma_{22} P^{2}-8\left(\sigma_{22}-\sigma_{11}\right) f_{20}\left(x_{1}\right)-i 4 \sigma_{12} f_{21}\left(x_{1}\right)\right] \\
\left(2 W_{2}-f_{2}\right)^{+}+\left(2 W_{2}-f_{2}\right)^{-}=4\left(\sigma_{22}-\sigma_{11}\right) g_{20}\left(x_{1}\right)+i 16 \sigma_{12} g_{21}\left(x_{1}\right),
\end{gathered}
$$


where

$$
\begin{aligned}
& f_{20}\left(x_{1}\right)=\left[\left(1-x_{1}^{2}\right) H_{1}^{\prime}\left(x_{1}\right)-x_{1} H_{1}\left(x_{1}\right)\right] P\left(x_{1}\right), \\
& f_{21}\left(x_{1}\right)=\left(4 x_{1}^{2}-1\right) P^{2}\left(x_{1}\right)-2 x_{1}\left(1-x_{1}^{2}\right) P\left(x_{1}\right) P^{\prime}\left(x_{1}\right), \\
& g_{20}\left(x_{1}\right)=-\left[\left(1-x_{1}^{2}\right) P\left(x_{1}\right) P^{\prime}\left(x_{1}\right)-2 x_{1} P^{2}\left(x_{1}\right)\right]\left(1-x_{1}^{2}\right), \\
& g_{21}\left(x_{1}\right)=\left(1-x_{1}^{2}\right) P\left(x_{1}\right) h_{1}^{\prime}\left(x_{1}\right) .
\end{aligned}
$$

The solutions to Eqs. (3.22) and (3.23) are

$$
\begin{gathered}
f_{2}(z)=-2\left(z^{2}-1\right)^{1 / 2}\left[\sigma_{22} P^{2}(z)+2\left(\sigma_{22}-\sigma_{11}\right) f_{20}(z)+i \sigma_{12} f_{21}(z)\right]+h_{2}(z), \\
2 W_{2}-f_{2}=2\left(\sigma_{22}-\sigma_{11}\right)\left[g_{20}(z)-H_{20}(z)\left(z^{2}-1\right)^{1 / 2}\right] \\
+i 8 \sigma_{12}\left[g_{21}(z)-H_{21}(z)\left(z^{2}-1\right)^{1 / 2}\right]
\end{gathered}
$$

where $h_{2}, H_{20}$, and $H_{21}$ are polynomials chosen so that both $f_{2}^{\prime}$ and $2 W_{2}^{\prime}-f_{2}^{\prime}$ tend to $O\left(1 / z^{2}\right)$ as $z$ tends to infinity. This completes the derivation for a three-term expansion. The determination of the unknown polynomials may be facilitated by the formulas provided in Appendix A.

The three-term expansion is now substituted into Eq. (2.22) to yield

$$
\begin{array}{r}
K_{1}-i K_{2}=\lim _{z \rightarrow 1} 2 \sqrt{2 \pi}(z-1)^{1 / 2} e^{-i \alpha_{c} / 2}\left\{W_{0}^{\prime}(z)+\varepsilon W_{1}^{\prime}(z)+\frac{1}{2} \varepsilon^{2} W_{2}^{\prime}(z)+\cdots\right\} \\
=e^{-i \alpha_{c} / 2} \sqrt{\pi}\left[\left(\sigma_{22}-i \sigma_{12}\right)+\varepsilon\left[2 P(1) \sigma_{12}-i H_{1}(1)\left(\sigma_{22}-\sigma_{11}\right)\right]\right. \\
+\frac{1}{2} \varepsilon^{2}\left\{-2 \sigma_{22} P^{2}(1)-2\left(\sigma_{22}-\sigma_{11}\right)\left[H_{20}(1)+2 f_{20}(1)\right]\right. \\
\left.\left.-i 2 \sigma_{12}\left[f_{21}(1)+4 H_{21}(1)\right]\right\}+\cdots\right],
\end{array}
$$

where

$$
\alpha_{c}=\operatorname{Tan}^{-1} \varepsilon Y^{\prime}(1)=\varepsilon \beta, \quad \beta=Y^{\prime}(1) .
$$

Expanding Eq. (3.30) in terms of $\varepsilon$, we finally obtain

$$
\begin{aligned}
\frac{K_{1}}{\sqrt{\pi}}= & \sigma_{22}-\varepsilon \sigma_{12}\left[\frac{\beta}{2}-2 P(1)\right] \\
& -\varepsilon^{2}\left\{\sigma_{22}\left[\frac{\beta^{2}}{8}+P^{2}(1)\right]+\left(\sigma_{22}-\sigma_{11}\right)\left[H_{20}(1)+2 f_{20}(1)+\frac{\beta}{2} H_{1}(1)\right]\right\}+\cdots,
\end{aligned}
$$

$$
\begin{aligned}
\frac{K_{2}}{\sqrt{\pi}}= & \sigma_{12}+\varepsilon\left[\frac{\beta}{2} \sigma_{22}+H_{1}(1)\left(\sigma_{22}-\sigma_{11}\right)\right] \\
& -\varepsilon^{2} \sigma_{12}\left[\frac{\beta^{2}}{8}-\beta P(1)-f_{21}(1)-4 H_{21}(1)\right]+\cdots .
\end{aligned}
$$

The circular-arc crack results are used to demonstrate the accuracy of the above explicit formulas. A circular-arc crack may be conveniently defined by

$$
x_{2}=\varepsilon Y\left(x_{1}\right)=-\frac{\varepsilon}{2}\left(1-x_{1}^{2}\right) \quad\left(\left|x_{1}\right|<1\right)
$$


where $2 \varepsilon$ is the central angle in radians sustained by the circular arc of radius $\varepsilon^{-1}$. It is also noted that the difference between Eq. (3.34) and the exact description for the circular arc is of the order of $\varepsilon^{3}$. Starting from Eq. (3.34) and using Eqs. (3.17), (3.20), (3.21), (3.24)-(3.33), together with Appendix A, we obtain

$$
\begin{gathered}
P\left(x_{1}\right)=-\frac{1}{2}, \quad h_{1}(z)=-\frac{1}{2} z^{2}+\frac{1}{4}, \quad H_{1}=\frac{1}{2} z, \\
f_{20}=\frac{1}{4}\left(2 x_{1}^{2}-1\right), \quad f_{21}=\frac{1}{4}\left(4 x_{1}^{2}-1\right), \\
g_{20}=g_{21}=\frac{1}{2} x_{1}\left(1-x_{1}^{2}\right), \\
h_{2}=2\left[\left(\sigma_{22}-\sigma_{11}\right)+i \sigma_{12}\right] z^{3}+\frac{1}{2}\left[4 \sigma_{11}-3 \sigma_{22}-i 3 \sigma_{12}\right] z, \\
H_{20}=H_{21}=-\frac{1}{2} z^{2}+\frac{1}{4}, \quad \beta=1,
\end{gathered}
$$

and

$$
\begin{aligned}
& \frac{K_{1}}{\sqrt{\pi}}=\sigma_{22}-\varepsilon \frac{3}{2} \sigma_{12}-\varepsilon^{2}\left[\frac{3}{8} \sigma_{22}+\frac{1}{2}\left(\sigma_{22}-\sigma_{11}\right)\right]+\cdots, \\
& \frac{K_{2}}{\sqrt{\pi}}=\sigma_{12}+\varepsilon\left[\frac{1}{2} \sigma_{22}+\frac{1}{2}\left(\sigma_{22}-\sigma_{11}\right)\right]-\varepsilon^{2} \frac{7}{8} \sigma_{12}+\cdots,
\end{aligned}
$$

where the last two expressions are identical to the three-term expressions deduced from the exact solution (Cotterell and Rice [4], Muskhelishvili [8]). The fact that these asymptotic formulas are accurate to $5 \%$ for circular-arc cracks with central angle less than $80^{\circ}$ suggests that the general formulas (3.32) and (3.33) may also be applied for

$$
\varepsilon<\frac{40}{180} \pi=0.698 .
$$

Moreover, the polynomial formulas provided in Appendix A may be straightforwardly summed, and hence the input polynomial $P\left(x_{1}\right)$ of Eq. (3.17) may be of any degree.

Holes with cusps $\left(Y_{+}-Y_{-} \geq 0\right)$. The functions $Y_{ \pm}$satisfy Eq.(2.17) so that the singularities at $z= \pm 1$ are still of the crack-tip type. However, it is rather difficult to treat this class of problems in completely general terms. The following specific problem is used to illustrate the procedure:

$$
Y_{+}\left(x_{1}\right)=\left(1-x_{1}^{2}\right)^{3 / 2}, \quad Y_{-}\left(x_{1}\right)=0 .
$$

The shape of such a regularly perturbed crack is depicted in Fig. 1(a). Using the above, we find from Eqs. (3.12) and (3.13) that

$$
\begin{aligned}
f_{1}^{+}-f_{1}^{-} & =\left(2 W_{1}-f_{1}\right)^{+}+\left(2 W_{1}-f_{1}\right)^{-} \\
& =i\left(\sigma_{22}-\sigma_{11}\right)\left(1-x_{1}^{2}\right)^{3 / 2}+i 2 \sigma_{12} x_{1}\left(1-x_{1}^{2}\right) .
\end{aligned}
$$

The following functions will appear in the upcoming solution:

$$
X(z)=\left(z^{2}-1\right)^{1 / 2}, \quad L(z)=\ln [(z-1) /(z+1)] .
$$




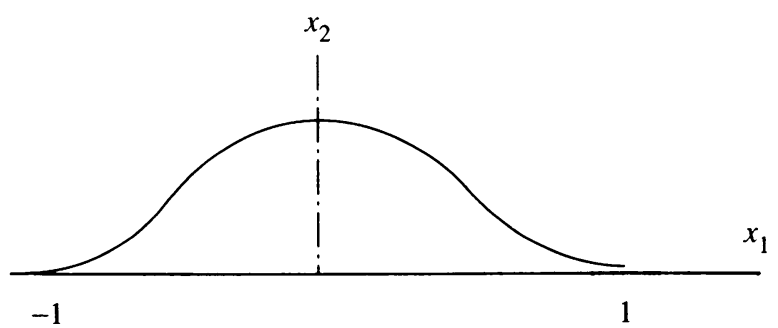

(a)

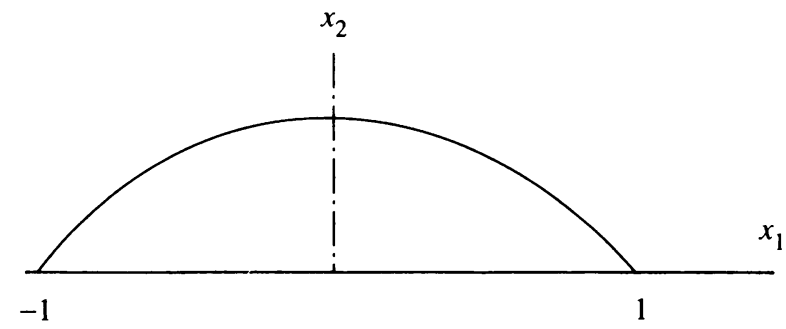

(b)

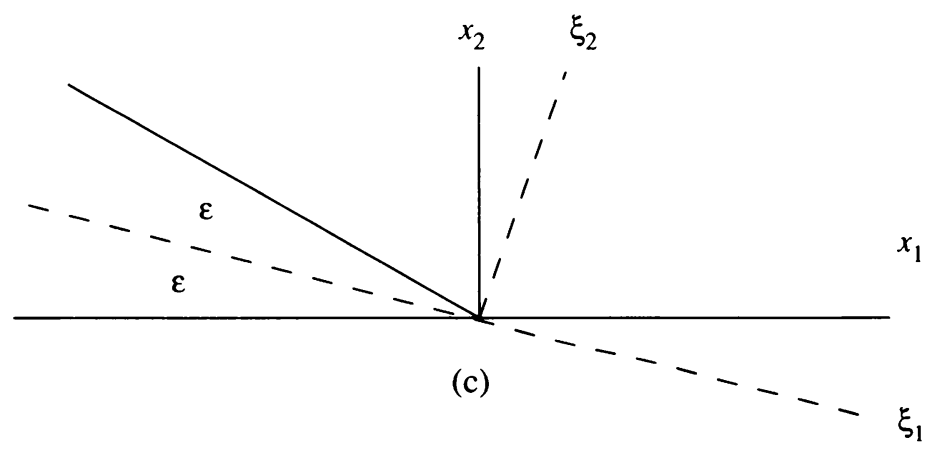

Fig. 1. (a) A regularly perturbed crack with cusps, (b) a singularly perturbed crack with notch tips, and (c) the magnified notch tip of (b).

They satisfy the conditions

$$
\begin{gathered}
X^{+}+X^{-}=0, \quad X^{+}-X^{-}=2 i\left(1-x_{1}^{2}\right)^{1 / 2} ; \\
L^{+}+L^{-}=2 \ln \left[\left(1-x_{1}\right) /\left(1+x_{1}\right)\right], \quad L^{+}-L^{-}=2 \pi i \\
(X L)^{+}+(X L)^{-}=-2 \pi\left(1-x_{1}^{2}\right)^{1 / 2}, \\
(X L)^{+}-(X L)^{-}=i 2\left(1-x_{1}^{2}\right)^{1 / 2} \ln \left[\left(1-x_{1}\right) /\left(1+x_{1}\right)\right] .
\end{gathered}
$$


Using Eqs. (3.42)-(3.44) and Eq. (3.40), we obtain

$$
\begin{aligned}
f_{1}(z)= & -\frac{1}{2}\left(\sigma_{22}-\sigma_{11}\right)\left[\left(z^{2}-1\right)^{3 / 2}-z^{3}+\frac{3}{2} z\right] \\
& -\frac{1}{\pi} \sigma_{12}\left[z\left(z^{2}-1\right) \ln (z-1) /(z+1)+2 z^{2}-\frac{4}{3}\right], \\
2 W_{1}-f_{1}= & \frac{i\left(\sigma_{22}-\sigma_{11}\right)}{2 \pi}\left[\left(z^{2}-1\right)^{3 / 2} \ln (z-1) /(z+1)+2 z\left(z^{2}-1\right)^{1 / 2}\right] \\
& -i \sigma_{12}\left[z\left(z^{2}-1\right)-\left(z^{2}-\frac{1}{2}\right)\left(z^{2}-1\right)^{1 / 2}\right],
\end{aligned}
$$

where homogeneous solutions have been added to ensure the vanishing of $f_{1}^{\prime}$ and $\left(2 W_{1}-f_{1}\right)^{\prime}$ at infinity (cf. Eqs. (3.20) and (3.21)). It follows from Eqs. (3.45) and (3.46) and Eq. (2.22) that the $O(\varepsilon)$-term of $K_{1}$ is zero. The second significant term of $K_{1}$, therefore, is governed by $W_{2}$.

Substituting all the known relations into Eqs. (3.15) and (3.16), we get

$$
\begin{aligned}
f_{2}^{+}-f_{2}^{-}= & \left(2 W_{2}-f_{2}\right)^{+}+\left(2 W_{2}-f_{2}\right)^{-} \\
= & \left(\sigma_{22}-\sigma_{11}\right)\left\{i \frac{6}{\pi} x_{1}\left(1-x_{1}^{2}\right)^{2} \ln \frac{1-x_{1}}{1+x_{1}}+i \frac{8}{\pi}\left(1-x_{1}^{2}\right)^{2} 6 x_{1}\left(1-x_{1}^{2}\right)^{2}\right. \\
& \left.\quad i \frac{4}{\pi} x_{1}^{2}\left(1-x_{1}^{2}\right)-i 3\left(2 x_{1}^{2}-1\right)\left(1-x_{1}^{2}\right)^{3 / 2}\right\} \\
& -i 2 \sigma_{22}\left(1-x_{1}^{2}\right)^{3 / 2} \\
& +\sigma_{12}\left\{i \frac{4}{\pi}\left(3 x_{1}^{2}-1\right)\left(1-x_{1}^{2}\right)^{3 / 2} \ln \frac{1-x_{1}}{1+x_{1}}+i 8 x_{1}\left(1-x_{1}^{2}\right)^{2}\right. \\
& \left.\quad-i 2 x_{1}\left(2 x_{1}^{2}-1\right)\left(1-x_{1}^{2}\right)+i \frac{24}{\pi} x_{1}\left(1-x_{1}^{2}\right)^{3 / 2}+2\left(6 x_{1}^{2}-1\right)\left(1-x_{1}^{2}\right)^{3 / 2}\right\} .
\end{aligned}
$$

The solutions for $W_{2}$ and $f_{2}$ may be obtained from the above by applying Eqs. (3.42)-(3.44). It may easily be checked that $f_{2}^{\prime}( \pm 1)=0$, and hence the possibility of a square-root singularity can only come from $2 W_{2}-f_{2}$. Moreover, only the two real terms in the above contribute to the value of $K_{1}$ (cf. Eq. (2.22)). Concentrating on this stated fact, we have

$$
\begin{aligned}
& \left(2 W_{2}-f_{2}\right)^{+}+\left(2 W_{2}-f_{2}\right)^{-} \\
& \quad=\left(\sigma_{22}-\sigma_{11}\right) 6 x_{1}\left(1-x_{1}^{2}\right)^{2}+\sigma_{12} 2\left(6 x_{1}^{2}-1\right)\left(1-x^{2}\right)^{3 / 2}+\cdots
\end{aligned}
$$

and

$$
\begin{aligned}
2 W_{2}-f_{2}= & 3\left(\sigma_{22}-\sigma_{11}\right)\left[z\left(1-z^{2}\right)^{2}-\left(z^{2}-1\right)^{1 / 2}\left(3 z^{4}-\frac{9}{2} z^{2}+\frac{9}{8}\right)\right] \\
& +\frac{\sigma_{12}}{\pi}\left[\left(6 z^{2}-1\right)\left(z^{2}-1\right)^{3 / 2} \ln \frac{z-1}{z+1}+\left(z^{2}-1\right)^{1 / 2}\left(12 z^{3}-10 z\right)\right]+\cdots,
\end{aligned}
$$

where $\cdots$ indicates the contribution of the imaginary terms of Eq. (3.47). Equation 
(2.22) is now used to yield

$$
\begin{aligned}
& \frac{K_{1}}{\sqrt{\pi}}=\sigma_{22}+\varepsilon^{2}\left[\frac{9}{32}\left(\sigma_{22}-\sigma_{11}\right)+\frac{1}{\pi} \sigma_{12}\right]+\cdots, \\
& \frac{K_{2}}{\sqrt{\pi}}=\sigma_{12}-\varepsilon\left[\frac{1}{\pi}\left(\sigma_{22}-\sigma_{11}\right)+\frac{1}{2} \sigma_{12}\right]+\cdots,
\end{aligned}
$$

which are the stress intensity factors associated with the crack configuration of Fig. $1(\mathrm{a})$.

4. Singularly perturbed cracks. Singularly perturbed cracks are defined by (cf. Eq. $(2.18))$

$$
Y_{+}^{\prime}( \pm 1) \neq Y_{-}^{\prime}( \pm 1)
$$

and, hence, are not cracks in that the singularities at $z= \pm 1$ are not of the squareroot type. Once again, it is difficult to discuss the class of problems in completely general terms. The following special choice suffices to illustrate the main difference between regularly and singularly perturbed cracks:

$$
Y_{+}\left(x_{1}\right)=\left(1-x_{1}^{2}\right), \quad Y_{-}\left(x_{1}\right)=0,
$$

which is depicted in Fig. 1(b). The singularities at $z= \pm 1$, therefore, are dictated by the notch angle $2(\pi-\varepsilon)$. While the notch angle may be approximated by $2 \pi$ for small values of $\varepsilon$, the mathematical singularities associated with small values of $\varepsilon$ are characteristically different from those associated with $\varepsilon=0$. It follows that the asymptotic series (3.1) and (3.2), developed for Eq. (4.2), cannot be valid for $z= \pm 1$ and therefore are termed an outer expansion.

Beginning with the reduced solution $f_{0}, W_{0}$ of Eqs. (3.8) and (3.9), we conclude from Eqs. (3.12) and (3.13) that $f_{1}$ and $W_{1}$ must satisfy the boundary conditions

$$
\begin{aligned}
f_{1}^{+}-f_{1}^{-} & =\left(2 W_{1}-f_{1}\right)^{+}+\left(2 W_{1}-f_{1}\right)^{-} \\
& =i\left(\sigma_{22}-\sigma_{11}\right)\left(1-x_{1}^{2}\right)+i 2 \sigma_{12} x_{1}\left(1-x_{1}^{2}\right)^{1 / 2} .
\end{aligned}
$$

It follows from the properties $(3.42)-(3.44)$ that

$$
\begin{aligned}
f_{1}(z)=-\frac{1}{2 \pi}\left(\sigma_{22}-\sigma_{11}\right) & {\left[\left(z^{2}-1\right) \ln \frac{z-1}{z+1}+2 z\right]+\sigma_{12}\left[z\left(z^{2}-1\right)^{1 / 2}-z^{2}+\frac{1}{2}\right], } \\
2 W_{1}-f_{1}= & -\frac{i}{2}\left(\sigma_{22}-\sigma_{11}\right)\left[\left(z^{2}-1\right)-z\left(z^{2}-1\right)^{1 / 2}\right] \\
& -\frac{i}{\pi} \sigma_{12}\left[z\left(z^{2}-1\right)^{1 / 2} \ln \frac{z-1}{z+1}+2\left(z^{2}-1\right)^{1 / 2}\right]
\end{aligned}
$$

which, together with Eqs. (3.8) and (3.9), constitute a two-term outer expansion. The function $W_{0}$ is square-root singular, and the functions $W_{1}$ and $f_{1}$ are both square-root and logarithmically singular. These are not the correct singular properties associated with a wedge of wedge angle $2(\pi-\varepsilon)$. Thus, the two-term expansions are not valid for $0 \leq|z \pm 1| \ll 1$.

To amplify the true singular character of the solution for $0 \leq|z-1| \ll 1$, a special inner expansion will be constructed. This expansion employs a dimensionless 
boundary-layer complex variable $\zeta$ defined by

$$
\zeta=\frac{z-1}{\varepsilon} e^{i \varepsilon}
$$

In terms of the polar representation, Fig. 1(c),

$$
\zeta=\xi_{1}+i \xi_{2}=\rho e^{i \psi}
$$

the region $0 \leq|z-1| \ll 1$ is a traction-free elastic wedge of wedge angle $2 \alpha=$ $2(\pi-\varepsilon)$, i.e.,

$$
-\alpha<\psi<\alpha, \quad \rho \geq 0 .
$$

Let us first examine the one-term symmetric and antisymmetric wedge-apex expansions associated with the above traction-free wedge. We begin by assuming

$$
\Omega(\zeta)=A^{+} \zeta^{1-\lambda}, \quad \phi(\zeta)=B^{+} \zeta^{1-\lambda} \text { or } \Omega(\zeta)=A^{-} \zeta^{1-\lambda}, \quad \phi(\zeta)=B^{-} \zeta^{1-\lambda}
$$

where $A^{ \pm}$and $B^{ \pm}$are real. The functions $\Omega$ and $\phi$ play, respectively, the roles of $W$ and $f$ in the new $\zeta$-plane. The vanishing of Eq. (2.9) along $\psi= \pm \alpha$ leads to the characteristic equation

$$
\operatorname{Sin} 2\left(1-\lambda^{( \pm)}\right) \alpha \pm\left(1-\lambda^{( \pm)}\right) \operatorname{Sin} 2 \alpha=0
$$

and the modal relation

$$
B^{ \pm}=A^{ \pm}\left[1+\operatorname{Cos} 2\left(1-\lambda^{( \pm)}\right) \alpha \pm\left(1-\lambda^{( \pm)}\right)(\operatorname{Cos} 2 \alpha-1)\right]
$$

where + and - are associated with the symmetric and antisymmetric deformations respectively.

The roots of Eq. (4.9) have been extensively studied in the literature (Williams [13]; England [5]; Moffatt and Duffy [7]). We find the summary information of Ting $[10,11]$ most complete. Let $\lambda=\lambda_{1}, \lambda_{2}, \ldots$ be the roots, ordered in such a way that $+1>\operatorname{Re} \lambda_{1}>\operatorname{Re} \lambda_{2}>\cdots$. Using the fact that $\pi-\alpha=\varepsilon$, we may easily deduce from Eq. (4.9) the following explicit results:

$$
\begin{aligned}
& \lambda_{1}^{(+)}=\frac{1}{2}-\frac{1}{4 \pi}(\pi-\alpha)^{3}+\cdots=\frac{1}{2}-\frac{1}{4 \pi} \varepsilon^{3}+\cdots \quad \text { (symmetric) }, \\
& \lambda_{1}^{(-)}=\frac{1}{2}-\frac{1}{\pi}(\pi-\alpha)+\cdots=\frac{1}{2}-\frac{1}{\pi} \varepsilon+\cdots \quad \text { (symmetric) },
\end{aligned}
$$

Thus, the one-term symmetric and antisymmetric modes are

$$
\begin{aligned}
& \left\{\begin{array}{l}
\Omega_{1}^{(+)}=\zeta^{1-\lambda_{1}^{(+)}} \\
\phi_{1}^{(+)}=-\frac{1}{2}(\pi-\alpha)^{2} \zeta^{1-\lambda_{1}^{(+)}}
\end{array}\right. \\
& \left\{\begin{array}{l}
\Omega_{1}^{(-)}=i \zeta^{1-\lambda_{1}^{(-)}} \\
\phi_{1}^{(-)}=i \frac{3}{2}(\pi-\alpha)^{2} \zeta^{1-\lambda_{1}^{(-)}} .
\end{array}\right.
\end{aligned}
$$

It is clear from Figs. 1(b), (c) that the desired inner expansion is neither symmetric nor antisymmetric with respect to the $\xi_{1}$-axis. 
The leading terms of the inner expansion must be of the form

$$
\begin{gathered}
W \sim \Omega(\zeta ; \varepsilon) \sim \Omega_{0}+A(\varepsilon) \Omega_{1}^{(+)}(\zeta)+B(\varepsilon) \Omega_{1}^{(-)}(\zeta)+\cdots, \\
f \sim \phi(\zeta ; \varepsilon) \sim 2 \Omega_{0}+A(\varepsilon) \phi_{1}^{(+)}(\zeta)+B(\varepsilon) \phi_{1}^{(-)}(\zeta)+\cdots
\end{gathered}
$$

where $\Omega_{0}$ is a constant associated with a rigid body displacement and $A(\varepsilon)$ and $B(\varepsilon)$ are real. The problem is considered to be solved if $\Omega_{0}, A(\varepsilon)$, and $B(\varepsilon)$ are determined. This can only be accomplished by applying the concept of matching (Cole [3]; Van Dyke [11]).

The outer-expansion variable $z$ and inner-expansion variable $\zeta$ are related by Eq. (4.6) which consists of a translation, a magnification, and a rotation. It is known that the complex functions $W, w$, and $f$ are not completely invariant under such transformations (Muskhelishrili [7]). For this reason we choose to match the two displacement fields directly.

For clarity and convenience we write

$$
z=x_{1}+i x_{2}=x+i y, \quad \zeta=\xi_{1}+i \xi_{2}=\xi+i \eta .
$$

Then

$$
\begin{aligned}
2\left(u_{x}+i u_{y}\right) & =\kappa W(z)-W(\bar{z})+(\bar{z}-z) \overline{W^{\prime}(z)}+f(\bar{z}), \\
2\left(u_{\xi}+u_{\eta}\right) & =\kappa \Omega(\zeta)-\Omega(\bar{\zeta})+(\bar{\zeta}-\zeta) \overline{\Omega^{\prime}(\zeta)}+\phi(\bar{\zeta}),
\end{aligned}
$$

where $[W, f]$ and $[\Omega, \phi]$ are, respectively, the outer and inner expansions. The following is a suitable matching variable:

$$
\zeta_{0}=\frac{z-1}{\varepsilon^{1 / 2}}
$$

It follows from Eqs. (4.19) and (4.6) that

$$
z=1+\varepsilon^{1 / 2} \zeta_{0}, \quad \zeta=\frac{\zeta_{0}}{\varepsilon^{1 / 2}} e^{i \varepsilon} .
$$

The required matching is to be conducted for $\zeta_{0}$ fixed and $\varepsilon \rightarrow 0$, i.e.,

$$
\left.\left.\left(u_{x}+i u_{y}\right)\right|_{z=1+\varepsilon^{1 / 2} \zeta_{0}} \sim\left(u_{\xi}+i u_{\eta}\right) e^{-i \varepsilon}\right|_{\zeta=e^{i \varepsilon} \zeta_{0} / \varepsilon^{1 / 2}}
$$

where the expressions on the two sides are defined by Eqs. (4.17) and (4.18). A simple substitution shows that to the order of $\varepsilon^{1 / 2}$ the left-hand side of Eq. (4.21) is dominated by $W_{0}(z)$ and $f_{0}(z)$, while the right-hand side is not affected by $\phi_{1}^{( \pm)}$. In fact, Eq. (4.21) is satisfied to the order of $\varepsilon^{1 / 2}$ if

$$
\left.\left.W_{0}(z)\right|_{z=1+\varepsilon^{1 / 2} \zeta_{0}} \sim \Omega(\zeta, \varepsilon)\right|_{\zeta=e^{i e} \zeta_{0} / \varepsilon^{1 / 2}}
$$

is satisfied to the order of $\varepsilon^{1 / 2}$. We have

$$
\begin{gathered}
\left.W_{0}\right|_{z=1+\varepsilon^{1 / 2} \zeta_{0}}=\frac{1}{2}\left[\frac{1}{2}\left(\sigma_{11}-\sigma_{22}\right)+i \sigma_{12}\right]+\varepsilon^{1 / 4} \frac{1}{\sqrt{2}}\left(\sigma_{22}-\sigma_{12}\right) \zeta_{0}^{1 / 2}+\cdots, \\
\left.\Omega\right|_{\zeta=e^{i \varepsilon} \zeta_{0} / \varepsilon^{1 / 2}}=\Omega_{0}+[A(\varepsilon)+i B(\varepsilon)] \frac{1}{\varepsilon^{1 / 4}} \zeta_{0}^{1 / 2}+\cdots,
\end{gathered}
$$


where the property

$$
\varepsilon^{-\varepsilon}=1-\varepsilon \ln \varepsilon+\cdots
$$

has been used in simplifying Eq. (4.24). Finally, we conclude from Eqs. (4.22)-(4.24) that

$$
\begin{gathered}
\Omega_{0}=\frac{1}{2}\left[\frac{1}{2}\left(\sigma_{11}-\sigma_{22}\right)+i \sigma_{12}\right] \\
A(\varepsilon)=\varepsilon^{1 / 2} \frac{1}{\sqrt{2}} \sigma_{22}, \quad B(\varepsilon)=-\varepsilon^{1 / 2} \frac{1}{\sqrt{2}} \sigma_{12} .
\end{gathered}
$$

The notch-tip singularity at $z=1$ is now completely defined to the order of $\varepsilon^{1 / 2}$.

We conclude this paper by emphasizing the simplicity and usefulness of Eqs. (3.41)-(3.44) in solving Cauchy and Hilbert problems.

Appendix A. Formulas for the unknown polynomials. The unknown polynomials are typified by the functions $h_{1}$ and $H_{1}$ of Eqs. (3.20) and (3.21) where $P$ is a given polynomial. Thus, it suffices to consider the dependence of $h_{1}$ and $H_{1}$ on a single term of $P$. We begin with the expansion

$$
\left(z^{2}-1\right)^{1 / 2}=z \sum_{n=0}^{\infty} b_{2 n} z^{-2 n} \quad\left(\left|z^{2}\right|>1\right)
$$

where

$$
\begin{gathered}
b_{0}=1, \quad b_{2}=-\frac{1}{2}, \quad b_{4}=-\frac{1}{8}, \quad b_{6}=-\frac{1}{16}, \quad b_{8}=-\frac{5}{128} \\
b_{10}=-\frac{7}{256}, \quad b_{2 n}=\frac{1}{2}\left(\frac{1}{2}-1\right) \cdots\left(\frac{1}{2}-n-1\right) / n ! .
\end{gathered}
$$

For a given one-term polynomial $Q(z)$, the condition

$$
Q(z)\left(z^{2}-1\right)^{1 / 2}-q(z) \rightarrow 0 \quad \text { as } z \rightarrow \infty
$$

is satisfied by

$$
\begin{gathered}
Q=C_{2 N} z^{2 N}, \quad q=C_{2 N} \sum_{m=0}^{N} b_{2(N-m)} z^{2 m+1} \\
Q=C_{2 N+1} z^{2 N+1}, \quad q=C_{2 N+1} \sum_{m=0}^{N+1} b_{2(N-m+1)} z^{2 m} .
\end{gathered}
$$

Similarly, the condition

$$
Q(z)-\left(z^{2}-1\right)^{1 / 2} q(z) \rightarrow \text { constant } \text { as } z \rightarrow \infty
$$

is satisfied by

$$
\begin{aligned}
& Q=C_{2 N} z^{2 N}, \quad q=\sum_{n=0}^{N-1} q_{2 n+1} z^{2 n+1} \\
& Q=C_{2 N+1} z^{2 N+1}, \quad q=\sum_{n=0}^{N} q_{2 n} z^{2 n}
\end{aligned}
$$


where

$$
\begin{aligned}
& q_{2 N}=C_{2 N+1}, \\
& q_{2 N-1}=C_{2 N}, \\
& q_{2 N-2}-\frac{1}{2} q_{2 N}=0, \\
& q_{2 N-3}-\frac{1}{2} q_{2 N-1}=0, \\
& q_{2 N-4}-\frac{1}{2} q_{2 N-2}-\frac{1}{8} q_{2 N}=0, \\
& q_{2 N-5}-\frac{1}{2} q_{2 N-3}-\frac{1}{8} q_{2 N-1}=0, \\
& \vdots \\
& q_{2}-\frac{1}{2} q_{4}-\frac{1}{8} q_{6}+\cdots+b_{2(N-1)} q_{2 N}=0, \\
& q_{1}-\frac{1}{2} q_{3}-\frac{1}{8} q_{5}+\cdots+b_{2(N-1)} q_{2 N-1}=0, \\
& q_{0}-\frac{1}{2} q_{2}-\frac{1}{8} q_{4}+\cdots+b_{2 N} q_{2 N}=0,
\end{aligned}
$$

and hence all $q_{m}$ 's may be straightforwardly determined.

\section{REFERENCES}

[1] L. T. Berezhnitskii and V. M. Sadivskii, Stress distribution near elastic inclusions with cuspidal points on the outline, Soviet Material Sci. 12, 261-265 (1976)

[2] L. T. Berezhnitskii and V. M. Sadivskii, Theory of sharp-pointed stress concentrations in anisotropic plates, Soviet Material Sci. 13, 303-311 (1977)

[3] J. D. Cole, Perturbation methods in applied mathematics, Blaisdell, Waltham, MA, 1968

[4] B. Cotterell and J. Rice, Slightly curved or kinked cracks, Internat. J. Fracture 16, 155-169 (1980)

[5] A. H. England, Complex Variable Methods in Elasticity, Wiley-Interscience, New York, 1971

[6] R. V. Goldstein and R. L. Salganik, Brittle fracture of solids with arbitrary cracks, Internat. J. Fracture 10, 507-423 (1974)

[7] H. K. Moffatt and B. R. Duffy, Local similarity solutions and their limitations, J. Fluid Mech. 96, 299-313 (1980)

[8] N. I. Muskhelishvili, Some Basic Problems of the Mathematical Theory of Elasticity, translated by J. R. M. Redok, Noordhoff, Groningen, 1953

[9] V. V. Panasynk and L. T. Berezhnitskii, Limit equilibrium of plates with sharp stress raisers, Soviet Material Sci. 1, 293-301 (1965)

[10] T. C. T. Ting, The wedge subjected to tractions: A paradox re-examined, J. Elasticity 14, 235-247 (1984)

[11] T. C. T. Ting, Asymptotic solution near the apex of an elastic wedge with curved boundaries, Quart. Appl. Math. 42, 467-476 (1985)

[12] M. Van Dyke, Perturbation Methods in Fluid Mechanics, Parabolic Press, Stanford, CA, 1975

[13] M. L. Williams, Stress singularities resulting from various boundary conditions in angular corners of plates in extension, J. Appl. Mech. 19, 526-528 (1952)

[14] R. A. Westman, Pressurized star crack, J. Math. Phys. 43, 191-198 (1964)

[15] C. H. Wu, Unconventional internal cracks Part 1: Symmetric variations of a straight crack, J. Appl. Mech. 40, 62-68 (1982)

[16] C. H. Wu, Unconventional internal cracks Part 2: Method of generating simple cracks, J. Appl. Mech. 49, 383-388 (1982)

[17] C. H. Wu, Stress and notch-stress concentration induced by slight depressions and protrusions, J. Appl. Mech. 60, 992-997 (1993) 